\title{
A CRITERION FOR DISFOCALITY
}

\author{
PUI-KEI WONG
}

ABstract. A linear differential equation of the second order with coefficients holomorphic in the unit disk is considered. It is shown that if the coefficients are $H^{p}$ functions such that their norms satisfy a certain inequality, then all nontrivial solutions of the equation will be disfocal in the disk.

In this note we are concerned with nontrivial solutions of the linear second order complex differential equation

$$
\left(w^{\prime}(z) / k(z)\right)^{\prime}+q(z) w(z)=0,
$$

where $k(z)$ and $q(z)$ are functions holomorphic in the open unit disk $D$ of the complex $z$-plane and $k(z) \neq 0$ there. According to Nehari [3] a nontrivial solution $w(z)$ of $(1)$ is said to be disfocal in $D$ if there do not exist two distinct points $\alpha$ and $\beta$ in $D$ such that $w(\alpha)=w^{\prime}(\beta)=0$. The equation (1) is said to be disfocal in $D$ if every nontrivial solution is disfocal in $D$. In the theorem below a sufficient condition for the disfocality of (1) is given when $k(z)$ and $q(z)$ are $H^{p}$ functions. To obtain boundary estimates in the proof we shall have need of an inequality of F. Carlson [1] which we quote below:

Lemma (CARLSON). Let $f(z)$ be holomorphic in $D$ and let $C$ be any rectifiable arc which lies in $D$. Let $\zeta$ be any point on the circle $|\zeta|=r$, $0<r<1$, which contains $C$ in its interior. Denote by

$$
V_{C}(\zeta)=\int_{C}|d(\arg (\zeta-z))|, \quad z \in C
$$

Then for any $p>0$,

$$
\int_{C}|f(z)| p|d z| \leqq \lim _{r \rightarrow 1} \frac{1}{\pi} \int_{0}^{2 \pi}\left|f\left(r e^{i \theta}\right)\right| p V_{C}\left(r e^{i \theta}\right) r d \theta,
$$

whenever the right-hand side has meaning.

We remark that when $p=2$ and $C$ is the real interval $(-1,1)$, inequality (2) reduces to the well-known Fejér-Riesz inequality. We shall take as the $H^{p}$ norm of $f$ :

Received by the editors November 23, 1970.

AMS 1970 subject classifications. Primary 34A20; Secondary 34C10.

Key words and phrases. Complex differential equations, disfocal solutions, $H^{p}$ functions. 


$$
\|f\|_{p}=\lim _{r \rightarrow 1}\left\{\int_{0}^{2 \pi}\left|f\left(r e^{i \theta}\right)\right| p_{r} d \theta\right\}^{1 / p} .
$$

ThEOREM. Let $k \in H^{\lambda}(D)$ and $q \in H^{\eta}(D)$, where $\lambda \geqq 1$ and $\eta \geqq 1$. If

$$
2^{2-\phi}\|k\|_{\lambda}\|q\|_{\eta}<1, \quad \phi=\lambda^{-1}+\eta^{-1},
$$

then equation (1) is disfocal in $D$.

Proof. Suppose the contrary; then there exist two points $\alpha \neq \beta$ in $D$ such that

$$
\left(w^{\prime}(z) / k(z)\right)^{\prime}+q(z) w(z)=0, \quad w(\alpha)=w^{\prime}(\beta)=0
$$

has a nontrivial solution $w(z)$. Following Nehari [2] we replace (4) by its equivalent integral equation. To this end we let $C$ denote the line segment $\{z=t \beta+(1-t) \alpha ; 0 \leqq t \leqq 1\}$. If $z$ is any point on $C$, we let $C_{1}$ and $C_{2}$ denote respectively the subarcs of $C$ joining $\alpha$ with $z$ and $z$ with $\beta$. Set

$$
K(z)=\int_{\alpha}^{z} k(\zeta) d \zeta
$$

and

$$
\begin{aligned}
G(z, \zeta) & =K(\zeta), & & \zeta \in C_{1}, \\
& =K(z), & & \zeta \in C_{2} .
\end{aligned}
$$

Then (4) is equivalent to

$$
w(z)=\int_{C} G(z, \zeta) q(\zeta) w(\zeta) d \zeta
$$

Let $z_{0}$ be a point of $C$ such that $\left|w\left(z_{0}\right)\right|=\max _{z \in C}|w(z)|$; then (5) implies

$$
1 \leqq \int_{C}\left|G\left(z_{0}, \zeta\right) q(\zeta) d \zeta\right|
$$

We note that for any line segment $C$ in $D$, there always exists an $r_{0}$, $0<r_{0}<1$, such that $C$ lies entirely in the disk of radius $r_{0}$. In particular this implies $V_{C}(\zeta) \leqq \pi$ for all circles $|\zeta|=r, r_{0}<r<1$. Hence by Carlson's inequality we have in this case the estimate

$$
\int_{C}|f(z)|^{p}|d z| \leqq\|f\|_{p}^{p}
$$

for every $f \in H^{p}(D)$. First suppose $\lambda>1$. Then Hölder's inequality 
together with (7) shows that for all $z \in C$,

$$
|K(z)| \leqq 2^{1 / \lambda^{\prime}}\|k\|_{\lambda}, \quad \lambda^{\prime}=(\lambda-1)^{-1} \lambda .
$$

When $\lambda=1$ the estimate (8) is again valid if we let $1 / \lambda^{\prime}=0$. Thus (8) holds for all $\lambda \geqq 1$ and $z \in C$. Putting this estimate into (6) one gets

$$
1 \leqq 2^{1 / \lambda^{\prime}}\|k\|_{\lambda} \int_{C}|q(\zeta) d \zeta|
$$

Again by Hölder's inequality and (7) we have

$$
\int_{C}|q(\zeta) d \zeta| \leqq 2^{1 / \eta^{\prime}}\|q\|_{\eta}
$$

where $\eta^{\prime}=(\eta-1)^{-1} \eta$. Combining this with (9) we arrive at

$$
1 \leqq 2^{2-\phi}\|k\|_{\lambda}\|q\|_{\eta}
$$

which is impossible in view of (3). This contradiction proves the Theorem.

\section{REFERENCES}

1. F. Carlson, Quelques inégalités concernant les fonctions analytiques, Ark. Mat. Astr. Fys. 29B (1943), no. 11, 1-6. MR 6, 205.

2. Zeev Nehari, On the zeros of solutions of second-order linear differential equations, Amer J. Math. 76 (1954), 689-697. MR 16, 131.

3. - Some function-theoretic aspects of linear second-order differential equations, J. Analyse Math. 18 (1967), 259-276. MR 35 \#4391.

Michigan State University, East Lansing, Michigan 48823 